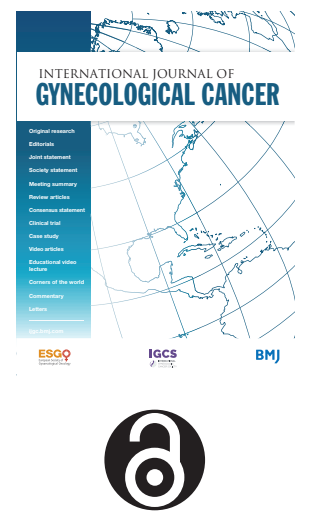

OPEN ACCESS

${ }^{1}$ Department of Public Health Programmes, Randers Regional Hospital, Randers, Denmark ${ }^{2}$ Department of Clinical Medicine, Aarhus University, Aarhus, Denmark ${ }^{3}$ Defactum, Central Denmark Region, Aarhus, Denmark ${ }^{4}$ Department of Pathology, Randers Regional Hospital, Randers, Denmark ${ }^{5}$ Department of Gynaecology, Randers Regional Hospital, Randers, Denmark

\section{Correspondence to}

Professor Berit Andersen, Department of Public Health Programmes, Randers Regional Hospital, Randers 8930 , Denmark; berand@rm.dk

Received 20 April 2020 Revised 10 September 2020 Accepted 11 September 2020 Published Online First 9 October 2020
Check for updates

(C) IGCS and ESG0 2020. Re-use permitted under CC BY-NC. No commercial re-use. Published by BMJ.

\begin{tabular}{l}
\hline To cite: Andersen B, \\
Njor SH, Jensen AMS, \\
et al. Int J Gynecol Cancer \\
2020;30:1678-1683. \\
\hline
\end{tabular}

\title{
HrHPV testing vs liquid-based cytology in cervical cancer screening among women aged 50 and older: a prospective study
}

\author{
Berit Andersen (D) ,1,2 Sisse Helle Njor, ${ }^{1,2}$ Anne Marie Schak Jensen, ${ }^{3}$ Tonje Johansen, ${ }^{4}$ \\ Ulla Jeppesen, ${ }^{5}$ Hans Svanholm ${ }^{1,4}$
}

\section{HIGHLIGHTS}

- HPV testing showed the best sensitivity for CIN2+/CIN3+ lesions but specificity was low.

- In HPV screening new triage method alternative to liquid-based cytology are highly warranted.

- The risk of overlooking gynecological cancers when shifting from cytology to HPV screening needs further consideration.

\section{ABSTRACT}

Introduction Evidence supports high-risk human papilloma virus (HPV) testing as the primary cervical cancer screening tool. However, benefits and harms should be carefully considered before replacing liquid-based cytology. In women age 50 and older, we evaluated how a commercially available DNA amplification HPV test compares with routine liquid-based cytology.

Methods This prospective study included 4043 patients who had a cervical sample analyzed from September 2011 to September 2012. Patients were followed between 64 and 76 months (median: 70 months). Samples were analyzed using both liquid-based cytology and the Cobas 4800 HPV DNA test. We calculated the diagnostic efficacy of liquid-based cytology and HPV, with or without the opposite test as triage, using cervical intraepithelial neoplasia (CIN2+/CIN3+) as reference.

Results The patients had a median age of 58 years, (range; 50-90). At baseline, HPV prevalence was 8.0\%: a total of $3.7 \%$ of patients had atypical squamous cells of undetermined significance or worse (ASCUS+). Positive test results were $1.9 \%$ for liquid-based cytology with HPV triage and $3.0 \%$ for HPV with liquid-based cytology triage. The cumulative incidence of CIN3+ was 1.0\% (40/4043). Sensitivities for CIN3+ were: liquid-based cytology $47.5 \%$ (31.5\%-63.9\%); liquid-based cytology with HPV triage 45.0\% (29.3\%-61.5\%); HPV 90.0\% (76.3\%-97.2\%); and HPV with liquid-based cytology triage $67.5 \%$ (50.9\%$81.4 \%)$. Corresponding specificities were: liquid-based cytology $96.6 \%$ (96.0\%-97.2\%); liquid-based cytology with HPV triage $98.5 \%$ (98.0\%-98.8\%); HPV 92.8\% (92.0\%-93.6\%); and HPV with liquid-based cytology triage $97.7 \%$ (97.2\%-98.1\%). At baseline, HPV testing overlooked five cases of gynecological cancer other than cervical cancer. Five cervical cancers were detected, two had been overlooked at baseline by liquid-based cytology and two by HPV testing

Conclusion HPV screening using DNA amplification is a promising alternative to liquid-based cytology in women age 50 and older, but evaluation of alternative triage methods is warranted. The risk of overlooking cancers needs consideration when replacing liquid-based cytology with HPV testing as a method for primary screening.

\section{INTRODUCTION}

For decades, the primary screening test of cervical cancer has been Pap smear, which has now largely been replaced by liquid-based cytology. Human papillomavirus (HPV) is recognized as a necessary agent for the development of most cervical cancers, and screening therefore often includes high-risk HPV testing. Some countries are shifting from primary microscopy to primary HPV testing. ${ }^{12}$

Randomized controlled trials have provided evidence to support the use of HPV testing as a primary screening tool in cervical cancer screening. ${ }^{3-10}$ However, these trials often used the hybrid capture 2 HPV DNA test and many compared the hybrid capture 2 test with Pap smears rather than liquid-based cytology. Hybrid capture 2 testing has been shown in a screening population to differ from tests such as COBAS, CLART, or ATPIMA, which are the preferred tests in most laboratories today. ${ }^{11}$ In a major trial from the USA (ATHENA study) HPV testing using COBAS had better performance than both liquid-based cytology alone, and co-testing with cytology and HPV testing. ${ }^{12}$

A recognized hurdle in primary HPV testing is reduced specificity and reduced positive predictive value, and possible triage of primary positive test results remains to be identified for all age groups. ${ }^{13}$ HPV testing may be especially favorable in older women, having the lowest HPV prevalence to avoid many unnecessary colposcopy referrals. Furthermore, in postmenopausal women, HPV screening may be favorable due to difficulties in distinguishing between atrophic changes and severe dysplasia at microscopy due to reduced estrogen levels. ${ }^{14}$

In this study on women age 50 and older, we aimed to estimate the diagnostic efficacy of liquid-based cytology, liquid-based cytology with HPV triage, HPV, and HPV with liquid-based cytology triage. 


\section{METHODS}

\section{Setting}

The study was conducted in the Central Denmark Region covering $23 \%$ of the Danish population. ${ }^{15}$ The target population for cervical cancer screening in the region is approximately 340000 women.

The Danish national guidelines for cervical cancer screening are met by all regions in the country. ${ }^{16}$ Between the ages of 23 to 64 , Danish women are invited every 3 (age 23-49) or 5 (age 50-64) years after their latest liquid-based cytology or their latest invitation, if no testing has been performed since. Up to two reminders are sent after 3 and 6 months to non-responders in all age groups. Women accepting the screening invitation book an appointment with their general practitioner who obtains a liquid-based cytology from the cervix and mails it to a pathology department for analysis. Women can also be tested opportunistically or as part of a control program. The overall testing coverage is approximately $75 \% .{ }^{17}{ }^{18} \mathrm{All}$ cervical cancer screening-related procedures are free of charge for the patient, including obtaining and analyzing samples, follow-up after positive test results, and treatment.

In the Central Denmark Region, all cervical cytology samples are routinely analyzed at the Department of Pathology, Randers Regional Hospital. The study protocol was presented to the Central
Denmark Region's Ethical Committee deciding that this was a study of laboratory methods and therefore obtaining informed consent from participants was not required (ref no. M-20110162). The study was approved by the Danish Data Protection Agency (ref no. 201141-6903 and 2017-41-5020).

\section{Population}

Samples from all women age 50 and older who had a cervical cytology sample analyzed at the Department of Pathology, Randers Regional Hospital, between 1 September 2011 and 14 September 2012 were included in the study.

We excluded women for whom the quality of the sample(s) did not allow proper microscopy or HPV analysis. If a woman had provided more than one sample in the inclusion period, the first adequate sample was categorized as the baseline sample. Any subsequent samples were categorized as follow-up samples.

\section{Handling of Baseline Samples}

All cytology samples were taken using liquid-based cytology (SurePathTM) and immediately mailed as per routine to the laboratory. On arrival, samples were divided into two parts, one for microscopy and one for HPV analysis. Samples for microscopy

\begin{tabular}{|c|c|c|c|c|c|}
\hline \multicolumn{6}{|l|}{ No. (\%) } \\
\hline Age at baseline (years) & $\begin{array}{l}50-54 \\
n=1138\end{array}$ & $\begin{array}{l}55-59 \\
n=1277\end{array}$ & $\begin{array}{l}60-64 \\
n=1218\end{array}$ & $\begin{array}{l}65+ \\
n=410\end{array}$ & $\begin{array}{l}\text { Total } \\
\mathrm{N}=4043\end{array}$ \\
\hline \multicolumn{6}{|l|}{ Baseline } \\
\hline \multicolumn{6}{|l|}{ Cytology alone } \\
\hline ASCUS+ & $70(6.2)$ & $34(2.7)$ & $35(2.9)$ & $15(3.7)$ & $154(3.8)$ \\
\hline ASCUS & $41(3.6)$ & $18(1.4)$ & $21(1.7)$ & $6(1.5)$ & $86(2.1)$ \\
\hline LSIL & $9(0.8)$ & $5(0.4)$ & $6(0.5)$ & $2(0.5)$ & $22(0.5)$ \\
\hline $\mathrm{HSIL}^{*}$ & $20(1.8)$ & $11(0.9)$ & $8(0.7)$ & $7(1.7)$ & $46(1.1)$ \\
\hline \multicolumn{6}{|l|}{ Cytology/HPV triage } \\
\hline Positive† & $36(3.2)$ & $20(1.6)$ & $13(1.1)$ & $10(2.4)$ & $79(2.0)$ \\
\hline \multicolumn{6}{|l|}{ HPV alone } \\
\hline HPV 16/18ł & $31(2.7)$ & $25(2.0)$ & $20(1.6)$ & $8(2.0)$ & $84(2.1)$ \\
\hline HrHPV other types only & $91(8.0)$ & $73(5.7)$ & $60(4.9)$ & $16(3.9)$ & $240(5.9)$ \\
\hline \multicolumn{6}{|l|}{ HPV/cytology triage } \\
\hline Positive*§ & $52(4.6)$ & $32(2.5)$ & $23(1.9)$ & $13(3.2)$ & $120(3.0)$ \\
\hline \multicolumn{6}{|l|}{ Follow-up } \\
\hline At least one histology & $225(19.8)$ & $188(14.7)$ & $148(12.2)$ & 79 (19.3) & $640(15.8)$ \\
\hline At least one cytology & $977(85.9)$ & $1098(86.0)$ & $462(37.9)$ & $279(68.0)$ & $2816(69.7)$ \\
\hline Total with follow-up result & $998(87.7)$ & $1124(88.0)$ & $506(41.5)$ & 303 (73.9) & $2931(72.5)$ \\
\hline Worst histology & $\mathrm{n}=225$ & $n=188$ & $n=148$ & $n=79$ & $n=640$ \\
\hline CIN2+ף & $25(11.1)$ & $18(9.6)$ & $15(10.1)$ & $6(7.6)$ & $64(10.0)$ \\
\hline $\mathrm{CIN} 3+^{* *}$ & $18(8.0)$ & $9(4.8)$ & $8(5.4)$ & $5(6.3)$ & $40(6.3)$ \\
\hline
\end{tabular}

Percentages are per column.

${ }^{*} \mathrm{HSIL}+$ included HSIL, AGC, adenoCIS, ASCH, and malignant cells from cervical cancer.

tincluding HSIL+ and ASCUS/LSIL with concurrent positive hrHPV test result (all hr-types).

$\$ 19$ women had both HPV 16/18 and other types.

§including all HPV 16/18 positive and hrHPV other types with concurrent ASCUS+.

ๆCIN2+ includes CIN2, CIN not specified, and diagnoses included in definition of CIN3+.

${ }^{* *} \mathrm{CIN} 3$ +includes CIN3, adenoCIS, AGC, and cervical cancer. 


\section{Original research}

were prepared and automatically pre-screened using a focal point slide profiler (BD diagnostics). ${ }^{19}$ The equipment identifies 10 areas of the slide of special interest. Hereafter, experienced cytotechnologists performed manual microscopy of the specimen while paying special attention to areas of interest. The Bethesda classification was used as per routine for establishing the diagnoses. ${ }^{20}$ The cytotechnologists were unaware of the HPV test result when performing the microscopy. Samples for HPV analysis were analyzed using the Cobas 4800 HPV DNA test (Roche Diagnostics GmBH, Switzerland) according to the manufacturer's protocols. The test identified HPV16, HPV18, and 12 other hrHPV types (31, 33, 35, 39, 45, 51, 52, $56,58,59,66$, and 68 ) in a single pool.

The physician, who obtained the sample, was notified by the laboratory about the results and received recommendations for follow-up according to national guidelines. ${ }^{19}$ Thus, women with samples positive for HPV 16/18, women with ASCUS/low-grade squamous intraepithelial lesions (LSIL) being HPV-positive (all HPV types), and women with adeno-CIS, ASCH, AGC, high-grade squamous intraepithelial lesions (HSIL), or malignant cells (from cervix or other) were recommended direct referral to colposcopy in a gynecological clinic. Women whose cytology results showed ASCUS in combination with an HPV-negative test, women with normal cytology who were HPV-positive for other types than 16/18, and women with LSIL were recommended control cytology after $6 / 12$ months. All other women were recommended screening after 5 years and received a screening invitation at that time if they were still in the screening age (50 to 64 years).

\section{Follow-up}

The population was followed until 31 December 2017. Thus, follow-up time was on average 5 years and 10 months.

Follow-up samples were tested according to routine guidelines. This was liquid-based cytology with HPV triage for all ages until 1 August 2014. From that date HPV with liquid-based cytology triage was used in women aged 60 to 64 . Histology samples were handled by experienced pathologists having gynaecologic pathology as their main competence, and according to current guidelines and classified into: CIN1, CIN2, CIN3, CIN not specified, adeno-CIS, cervical cancer, gynaecologic cancer not cervical cancer, benign tumor, cyst, or within normal limits. ${ }^{21}$

\section{Handling of Data}

All data on microscopy, HPV testing, and histology samples were registered into the Danish Pathology Data Bank using the unique civil personal registration number. The civil personal registration number was used for linking baseline data with follow-up data. ${ }^{22}$

Participants were stratified by age (5-year intervals). Baseline testing was reported using the following definitions of positive testing: liquidbased cytology alone, a positive result was defined as ASCUS+; liquidbased cytology with HPV triage,a positive test was HSIL +or ASCUS/ LSIL with concurrent positive HPV test result (all HPV types); HPV alone, a positive result included all hrHPV types; and HPV testing with liquidbased cytology triage, we categorized a positive test result as either HPV 16/18 positive or positive for other hrHPV types with concurrent ASCUS+. For follow-up, the worst histological diagnosis was categorized into CIN3+ (including CIN3, adeno-CIS, and cervical cancer), CIN2+ (including CIN2, CIN not specified and diagnoses included in definition of CIN3+), or no significant abnormality (including CIN1, cyst, benign tumor, and malignancy not from cervix).

Baseline results using the two testing procedures (liquid-based cytology and HPV testing) were compared. Furthermore, the baseline results were compared with the worst histology diagnosis from the cervix in the follow-up period. Finally, we calculated sensitivity (baseline-positive cases/all CIN2+ or CIN3+ cases), specificity (baseline-negative cases/all cases without subsequent CIN2 +or CIN3+), positive predictive value (baseline positive cases with subsequent CIN2+ or CIN3+/all baseline positive cases), and negative predictive value (baseline negative cases without subsequent CIN2 or CIN3+/all baseline negative cases with $95 \%$ confidence intervals). These figures were calculated for liquid-based cytology, liquid-based cytology with HPV triage, HPV, and HPV with liquidbased cytology triage.

Statistical analyzes were performed using StataCorp LLC, release 15.

\section{RESULTS}

A total of 4043 women had at least one adequate sample in the baseline period. Of these, $3694(91.4 \%)$ samples were obtained in general practice, while the remaining were obtained in a gynecology department or at a private gynecology practice. In the follow-up period, a total of 5476 samples (cytological or histological) were registered from women in the study population: 640 women (15.8\%) had at least one histology sample and 2816 women (69.7\%) had at least one additional cytology sample. The highest follow-up rates were seen among women aged 50-54 (87.8\%) and 55-59 (88.0\%) at baseline, reflecting that these women were re-invited to screening after 5 years (Table 1). At baseline, liquid-based cytology showed ASCUS+ in 154 cases (3.8\%; 154/4,043): the HPV positivity rate was $8.0 \%(324 / 4,043)$, of which $2.1 \%(84 / 4,043)$ were HPV $16 / 18$ positive and $5.9 \%(240 / 4,043)$ were positive only for other hrHPV types. The cumulative incidence of CIN2+ was 1.6\% (64/4043): the cumulative incidence of CIN3 +1.0\% (40/4043) (Table 1).

Table 2 shows the correlation between liquid-based cytology and HPV at baseline. A total of 66 of 84 HPV 16/18-positive samples were within normal limits in the liquid-based cytology, while 25 of $46 \mathrm{HSIL}$ +diagnosesand five cases of malignancies not from the cervix diagnosed at baseline were HPV-negative at baseline.

Table 3 shows that of the 64 CIN2 +cases, 30 were predicted by use of liquid-based cytology alone, 27 were predicted if HPV testing was added as a triage, 53 were predicted by the use of HPV testing alone, and 35 cases were predicted after adding liquid-based cytology triage to primary HPV testing. Of the $40 \mathrm{CIN} 3+$ cases, 19 were predicted by the use of liquid-based cytology alone, 18 were predicted if HPV testing was added as a triage, 36 were predicted by HPV testing alone, and 27 cases were predicted after adding liquid-based cytology triage to primary HPV testing.

Table 4 shows the diagnostic efficacy of the testing strategies. Sensitivity for CIN3 + was highest for HPV testing without triage (90.0\% $(76.3 \%-97.2 \%))$ and lowest for liquid-based cytology with HPV triage $(45.0 \%(29.3 \%-61.5 \%))$. On the contrary, specificity was highest for liquid-based cytology with HPV triage (98.5\% (98.0\%-98.8\%)) and lowest for HPV testing without triage $(92.8 \%(92.0 \%-93.6 \%))$. Similar patterns were seen using $\mathrm{CIN} 2+$ as the reference.

Five of the CIN2+ cases were cervical cancers (data not shown). Two of these were within normal limits at baseline in liquid-based 
Table 2 Correlation between liquid-based cytology and HPV result at baseline $(n=4043)$

\begin{tabular}{|c|c|c|c|c|}
\hline \multirow{2}{*}{$\begin{array}{l}\text { Baseline } \\
\text { Liquid-based cytology }\end{array}$} & \multicolumn{4}{|c|}{$\begin{array}{l}\text { Baseline HPV } \\
\text { No. (\%) }\end{array}$} \\
\hline & HPV 16/18 & hrHPV other types* & hrHPV- negative & Total \\
\hline ASCUS & $5(6.0)$ & $15(6.3)$ & $66(1.8)$ & $86(2,1)$ \\
\hline LSIL & $4(4.8)$ & $9(3.8)$ & $9(0.2)$ & $22(0.5)$ \\
\hline HSIL+† & $9(10.7)$ & $12(5.0)$ & $25(0.7)$ & $46(1.1)$ \\
\hline No cervix abnormality $\ddagger$ & $66(78.6)$ & $204(85.0)$ & 3619 (97.3) & 3889 (96.2) \\
\hline Total & $84(100)$ & $240(100)$ & $3719(100)$ & $4043(100)$ \\
\hline
\end{tabular}

Percentages are per column.

*excluding 19 having both HPV 16/18 and other types.

†HSI+ included HSIL, AGC, adenoCIS, ASCH, and malignant cells from cervical cancer.

łIncluding five patients with malignancies not from cervix (all were hrHPV negative).

cytology and two others were HPV negative at baseline. Furthermore at baseline, liquid-based cytology detected five gynecological cancers other than cervical cancer, all of which were HPV-negative.

\section{DISCUSSION}

In this study of more than 4000 women aged 50 or older tested in the Danish cervical cancer screening program, we found that HPV testing without triage had a significantly better sensitivity for detecting CIN2+ and CIN3+ lesions than all other algorithms. Concurrently, specificity was significantly lower than that of all other algorithms. The use of HPV testing with liquid-based cytology triage improved specificity and, although sensitivity was concurrently reduced, it was still better than liquid-based cytology with or without HPV triage.
This study takes advantage of national routine registration of all cervical samples and data storage in a national register with high validity. ${ }^{22}$ Further, three out of four women had at least one sample in the follow-up period, and follow-up reached almost $90 \%$ among those still of screening age after their baseline testing. Among women aged $65+$ at baseline, follow-up was $68.1 \%$, which is probably explained by the one-time offer of an HPV test in 2017 to all women born before 1948 (being $69+$ in 2017). ${ }^{23}$ Finally, the usefulness of the study and the results in Danish and other settings is underlined by the fact that Cobas 4800 and liquid-based cytology, are still among the most used tests in laboratories around the world.

We do acknowledge that we do not know exactly why women were tested at baseline or follow-up, nor do we have data on histology indications, as such information is not registered routinely. Another limitation is that women aged $60-64$ at baseline

Table 3 Comparison of baseline test results and subsequent diagnosis in the follow-up period 2 ( $n=4043$ )

\begin{tabular}{|c|c|c|c|c|c|}
\hline & \multicolumn{5}{|c|}{ Worst histology diagnosis $\mathrm{n}(\%)$} \\
\hline & CIN2+ n=64 & CIN3+n=40 & $\begin{array}{l}\text { No histological } \\
\text { abnormality } n=576\end{array}$ & $\begin{array}{l}\text { No histology } \\
n=3403\end{array}$ & Total $n=4043$ \\
\hline \multicolumn{6}{|l|}{ Baseline } \\
\hline \multicolumn{6}{|c|}{ Cytology alone } \\
\hline ASCUS+ & $30(46.9)$ & $19(47.5)$ & $95(16.5)$ & $29(0.9)$ & $154(3.8)$ \\
\hline ASCUS & 7 (10.9) & $5(12.5)$ & $54(9.4)$ & $25(0.7)$ & $86(2.1)$ \\
\hline LSIL & $5(7.8)$ & $4(10.0)$ & $14(2.4)$ & $3(0.1)$ & $22(0.5)$ \\
\hline $\mathrm{HSIL}+{ }^{*}$ & $18(28.1)$ & $10(25.0)$ & $27(4.7)$ & $1(0.0)$ & $46(1.1)$ \\
\hline \multicolumn{6}{|c|}{ Cytology/HPV triage } \\
\hline Positive† & $27(42.2)$ & $18(45.0)$ & $49(8.5)$ & $3(0.1)$ & $79(2.0)$ \\
\hline \multicolumn{6}{|l|}{ HPV alone } \\
\hline HPV 16/18 & $18(28.1)$ & $14(35.0)$ & $57(9.9)$ & $9(0.3)$ & $84(2.1)$ \\
\hline HrHPV other & $35(54.7)$ & $22(55.0)$ & $103(17.9)$ & $102(3.0)$ & $240(5.9)$ \\
\hline \multicolumn{6}{|c|}{ HPV/cytology triage } \\
\hline Positiveł & $35(54.7)$ & $27(67.5)$ & 75 (13.0) & $10(0.3)$ & $120(3.0)$ \\
\hline
\end{tabular}

Percentages are per column.

*HSIL+ included HSIL, AGC, adenoCIS, ASCH, and malignant cells from cervical cancer.

†positive includes referrals to colposcopy: HSIL+, and ASCUS/LSIL with concurrent positive hrHPV test result (all hr-types).

łpositive includes referrals to colposcopy: all HPV 16/18 positive and hrHPV other types positive with concurrent ASCUS+. 


\section{Original research}

Table 4 Diagnostic efficacy of liquid-based cytology, liquid-based cytology with HPV triage, HPV, and HPV with liquid-based cytology triage, using CIN2+ and CIN3+ as reference $(n=4043)$

\begin{tabular}{|c|c|c|c|c|}
\hline & \multicolumn{4}{|c|}{$\begin{array}{l}\text { Diagnostic test at baseline } \\
\%(95 \% \mathrm{Cl})\end{array}$} \\
\hline & Liquid-based cytology & $\begin{array}{l}\text { Liquid-based cytology } \\
\text { +HPV triage }\end{array}$ & HPV & $\begin{array}{l}\text { HPV+ liquid-based } \\
\text { cytology triage }\end{array}$ \\
\hline \multicolumn{5}{|l|}{ Reference: CIN2+ } \\
\hline Sensitivity & $\begin{array}{l}46.9 \%(30 / 64) \\
(34.3 \% \text { to } 59.8 \%)\end{array}$ & $\begin{array}{l}42.2 \%(27 / 64) \\
(29.9 \% \text { to } 55.2 \%)\end{array}$ & $\begin{array}{l}82.8 \%(53 / 64) \\
(71.3 \% \text { to } 91.1 \%)\end{array}$ & $\begin{array}{l}54.7 \%(35 / 64) \\
(41.7 \% \text { to } 67.2 \%)\end{array}$ \\
\hline Positive predictive value & $\begin{array}{l}19.5 \%(30 / 154) \\
(13.5 \% \text { to } 26.6 \%)\end{array}$ & $\begin{array}{l}34.2(27 / 79) \\
(23.9 \% \text { to } 45.7 \%)\end{array}$ & $\begin{array}{l}16.4 \%(53 / 324) \\
(12.5 \% \text { to } 20.8 \%)\end{array}$ & $\begin{array}{l}29.2 \%(35 / 120) \\
(21.2 \% \text { to } 38.2 \%)\end{array}$ \\
\hline Negative predictive value & $\begin{array}{l}99.1(3,855 / 3,889) \\
(98.8 \% \text { to } 99.4 \%)\end{array}$ & $\begin{array}{l}99.1 \%(3,927 / 3,964) \\
(98.7 \% \text { to } 99.3 \%)\end{array}$ & $\begin{array}{l}99.7 \%(3,708 / 3,719) \\
(99.5 \% \text { to } 99.9 \%)\end{array}$ & $\begin{array}{l}99.3 \%(3,894 / 3,923) \\
(98.9 \% \text { to } 99.5 \%)\end{array}$ \\
\hline \multicolumn{5}{|l|}{ Reference: CIN3+ } \\
\hline Positive predictive value & $\begin{array}{l}12.3 \%(19 / 154) \\
(7.6 \% \text { to } 18.6 \%)\end{array}$ & $\begin{array}{l}22.8 \%(18 / 79) \\
(14.1 \% \text { to } 33.6 \%)\end{array}$ & $\begin{array}{l}11.1 \%(36 / 324) \\
(7.9 \% \text { to } 15.0 \%)\end{array}$ & $\begin{array}{l}22.5 \%(27 / 120) \\
(15.4 \% \text { to } 31.0 \%)\end{array}$ \\
\hline Negative predictive value & $\begin{array}{l}99.5 \%(3,868 / 3,889) \\
(99.2 \% \text { to } 99.7 \%)\end{array}$ & $\begin{array}{l}99.4 \%(3,942 / 3,964) \\
(99.2 \% \text { to } 99.7 \%)\end{array}$ & $\begin{array}{l}99.9 \%(3,715 / 3,719) \\
(99.7 \% \text { to } 100.0 \%)\end{array}$ & $\begin{array}{l}99.7 \%(3,910 / 3,923) \\
(99.4 \% \text { to } 99.8 \%)\end{array}$ \\
\hline
\end{tabular}

were neither routinely invited again for screening nor covered by the one-time screening offer. ${ }^{23}$

We found an overall HPV prevalence at baseline of $8.0 \%$, which was highest $(10.7 \%)$ among the youngest women $(50-54$ years) and lowest $(5.8 \%)$ among the oldest women $(65+)$. The figures for the youngest women were slightly higher than those reported in another Danish study of similar age groups from another area (Capital Region of Denmark) a few years' earlier (2002-2005). ${ }^{24}$ In this earlier study, the HPV prevalence was $8.7 \%$ for women aged $45-54,7.4 \%$ for women aged $55-64 \%$, and $5.7 \%$ for women $65+$. These minor differences may be due to differences between areas, differences in diagnostic test methods used as demonstrated in a study from Copenhagen, or a general rise in the spreading of the infection in these age groups in Denmark. ${ }^{13}$

Replacing liquid-based cytology screening by HPV screening deserves a thorough evaluation of benefits and harms. ${ }^{18}$ The main benefit of HPV screening is increased sensitivity for detecting severe dysplasia which, expectedly, will reduce the cervical cancer incidence. In our study, the sensitivity for CIN3+ almost doubled (from $47.5 \%$ to $90.0 \%$ ) if liquid-based cytology alone was replaced by HPV alone. However, the specificity declined significantly (from $96.0 \%$ to $92.8 \%$ ), which will affect a relatively large number of women. Adding liquid-based cytology triage to HPV screening improved the specificity to $97.7 \%$, which is more acceptable, however, at the same time, the sensitivity declined to $67.5 \%$. It could be speculated that this is due to not yet established cytology changes that will follow the HPV infection or due to specimens not being representative. Whatever the reasons, our findings underline that the diagnostic efficacy of a sequence of tests is only as good as the most deficient single analysis. New triage methods such as p16/Ki-67 dual-stained cytology could be a future alternative. ${ }^{25}$
Referring screen positive women without dysplasia for gynecologic evaluation should be minimized as it is costly and may constitute an unnecessary burden for the women and the healthcare system. In our study, positive rates rose from $1.9 \%$ in liquid-based cytology with HPV triage to $3.0 \%$ in HPV with liquid-based cytology as triage. This is in opposition to a recent Swedish evaluation of similar test algorithms in women aged 56-60 where the referral rate was the lowest in HPV with liquid-based cytology triage.$^{26} \mathrm{~A}$ lower HPV prevalence of $5.5 \%$ in the Swedish study population compared with $8.1 \%$ in our population may partly explain this difference. A Canadian study (HPV FOCAL) had a rather high referral rate to gynecological examination compared with our setting, and the referrals doubled in hybrid capture 2 testing with liquid-based cytology as a triage compared with liquid-based cytology with hybrid capture 2 testing as a triage $\left(58.9 / 1,000\right.$ and $30.0 / 1,000$, respectively) ${ }^{6}$ The explanation for this is not straightforward. The prevalence in HPV FOCAL was $8.2 \%$ and similar to the prevalence reported in our study, but the Canadian study reported on the entire age span from 25 to 65 years. Nevertheless, alternative methods for triaging should be considered in the future to maintain high sensitivity and increase specificity, simultaneously. ${ }^{1327}$

We found that five cancer cases from other sites than the cervix were identified by microscopy, but they were all HPV-negative. It is not expected to identify other cancers, and it can be argued that identification of non-cervix cancers is not the purpose of cervical cancer screening, still, earlier diagnosis may be a positive side effect that will disappear when switching to HPV screening.

Two cases of cervical cancer were not predicted by liquid-based cytology and another two cases of cervical cancer were not predicted by HPV test at baseline. We do not know the details of these cancers, but it could be speculated that the liquid-based cytology-negative 
cervical cancer cases were adenocarcinomas, corresponding to the fact that the incidence of squamous cell carcinoma has declined over the past decades, which is not the case for adenocarcinomas. ${ }^{28} \mathrm{~A}$ recent Swedish study showed that HPV-negative cervical cancer has a poorer prognosis than HPV-positive cervical cancer. ${ }^{29}$ These findings underline the preventive purpose of the screening program and the need for continuous testing at regular intervals to reduce the burden of cervical cancer in the population.

In conclusion, HPV screening using DNA amplification is a promising alternative to liquid-based cytology in women aged 50 or older because of its much higher sensitivity. However, even in this age group of women with a relatively low HPV prevalence, the specificity is concurrently significantly reduced. Adding liquid-based cytology as a triage increases the specificity, but at the same time significantly reduces sensitivity. We do not yet know the optimal strategy if we shift from liquid-based cytology to HPV screening in an otherwise wellfunctioning cervical cancer screening program. The significance of overlooking cervical cancers or non-cervical cancers in HPV screening needs further evaluation.

Contributors BA contributed to the study concept and design, interpretation of data, and drafed the manuscript. SHN contributed to interpretation of data and made critical revision of the manuscript for important intellectual content. AMSJ participated in the analysis and interpretation of data, and made critical revision of the manuscript for important intellectual content. TJ contributed in the study design and with critical revision of the manuscript for important intellectual content. UJ contributed to the study concept and design with gynecological expertise, and made critical revision of the manuscript for important intellectual content. HS made the study concept and design, participated in the acquisition, analysis and interpretation of data, and made critical revision of the manuscript for important intellectual content.

Funding Diagnostic kits in this study were provided free of charge by Roche Diagnostics.

Disclaimer Roche Diagnostics had no role in the design, collection of data, statistical analyses, interpretation of results, or writing of the manuscript.

Competing interests The study received diagnostic kits free of charge from Roche Diagnostics. BA has previously received test kits from Roche and Axlab for other studies.

Patient consent for publication Not required.

Provenance and peer review Not commissioned; externally peer reviewed.

Data availability statement No data are available. According to Danish law, data can only be made available on an aggregated level after approval by the Danish authorities.

Open access This is an open access article distributed in accordance with the Creative Commons Attribution Non Commercial (CC BY-NC 4.0) license, which permits others to distribute, remix, adapt, build upon this work non-commercially, and license their derivative works on different terms, provided the original work is properly cited, an indication of whether changes were made, and the use is noncommercial. See: http://creativecommons.org/licenses/by-nc/4.0/.

ORCID iD

Berit Andersen http://orcid.org/0000-0003-4074-6504

\section{REFERENCES}

1 Walboomers JM, Jacobs MV, Manos MM, et al. Human papillomavirus is a necessary cause of invasive cervical cancer worldwide. J Pathol 1999;189:12-19.

2 Wentzensen N, Arbyn M, Berkhof J, et al. How HPV knowledge is changing screening practice. Int J Cancer 2017;15:2192-200.

3 Ronco G, Dillner J, Elfström KM, et al. Efficacy of HPV-based screening for prevention of invasive cervical cancer: follow-up of four European randomised controlled trials. Lancet 2014;383:524-32.
4 Leinonen MK, Nieminen P, Lönnberg S, et al. Detection rates of precancerous and cancerous cervical lesions within one screening round of primary human papillomavirus DNA testing: prospective randomised trial in Finland. BMJ 2012;345:e7789.

5 Ronco G, Giorgi-Rossi P, Carozzi F, et al. Efficacy of human papillomavirus testing for the detection of invasive cervical cancers and cervical intraepithelial neoplasia: a randomised controlled trial. Lancet Oncol 2010;11:249-57.

6 Ogilvie GS, Krajden M, van Niekerk D, et al. HPV for cervical cancer screening (HPV FOCAL): complete round 1 results of a randomized trial comparing HPV-based primary screening to liquid-based cytology for cervical cancer. Int J Cancer 2017;140:440-8.

7 Sankaranarayanan R, Nene BM, Shastri SS, et al. HPV screening for cervical cancer in rural India. N Engl J Med 2009;360:1385-94.

8 Kitchener HC, Almonte M, Thomson C, et al. HPV testing in combination with liquid-based cytology in primary cervical screening (ARTISTIC): a randomised controlled trial. Lancet Oncol 2009;10:672-82.

9 Naucler P, Ryd W, Tornberg S, et al. Human papillomavirus and Papanicolou tests to screen for cervical cancer. N Engl J Med 2007;18:1589-97.

10 Rijkaart DC, Berkhof J, Rozendaal L, et al. Human papillomavirus testing for the detection of high-grade cervical intraepithelial neoplasia and cancer: final results of the POBASCAM randomised controlled trial. Lancet Oncol 2012;13:78-88.

11 Rebolj M, Bonde J, Preisler S, et al. Human papillomavirus assays and cytology in primary cervical screening of women aged 30 years and above. PLoS One 2016;11:e0147326.

12 Wright TC, Stoler MH, Behrens CM, et al. Primary cervical cancer screening with human papillomavirus: end of study results from the ATHENA study using HPV as the first-line screening test. Gynecol Oncol 2015;136:189-97.

13 Cuschieri K, Ronco G, Lorincz A, et al. Eurogin roadmap 2017: triage strategies for the management of HPV-positive women in cervical screening programs. Int J Cancer 2018;143:735-45.

14 Bateson DJ, Weisberg E. An open-label randomized trial to determine the most effective regimen of vaginal estrogen to reduce the prevalence of atrophic changes reported in postmenopausal cervical smears. Menopause 2009;16:765-9.

15 Statistics Denmark. Available: https://www.dst.dk/en [Accessed 14 Feb 2020].

16 Danish Health Authority. (guidelines in Danish). Available: https:// www.sst.dk [Accessed 14 Feb 2020].

17 Tranberg M, Larsen MB, Mikkelsen EM, et al. Impact of opportunistic testing in a systematic cervical cancer screening program: a nationwide registry study. BMC Public Health 2015;15:681.

18 Lynge E, Andersen B, Christensen J, et al. Cervical screening in Denmark - a success followed by stagnation. Acta Oncol 2018;57:354-61.

19 Chute DJ, Lim H, Kong CS. BD focalpoint slide profiler performance with atypical glandular cells on SurePath Papanicolaou smears. Cancer Cytopathol 2010;118:68-74.

20 Solomon D, Davey D, Kurman R, et al. The 2001 Bethesda system: terminology for reporting results of cervical cytology. JAMA 2002;287:2114-9.

21 Richart RM. Cervical intraepithelial neoplasia. Pathol Annu 1973;8:301-28.

22 Bjerregaard B, Larsen OB. The Danish pathology register. Scand J Public Health 2011;39:72-4.

23 Andersen B, Christensen BS, Christensen J, et al. HPV-prevalence in elderly women in Denmark. Gynecol Oncol 2019;154:118-23.

24 Kjær SK, Munk C, Junge J, et al. Carcinogenic HPV prevalence and age-specific type distribution in 40,382 women with normal cervical cytology, ASCUS/LSIL, HSIL, or cervical cancer: what is the potential for prevention? Cancer Causes Control 2014;25:179-89.

25 Wright TC, Behrens CM, Ranger-Moore J, et al. Triaging HPV-positive women with $\mathrm{p} 16 / \mathrm{Ki}-67$ dual-stained cytology: results from a sub-study nested into the ATHENA trial. Gynecol Oncol 2017;144:51-6.

26 Lamin H, Eklund C, Elfström KM, et al. Randomised healthcare policy evaluation of organised primary human papillomavirus screening of women aged 56-60. BMJ Open 2017;7:e014788.

27 Clarke MA, Cheung LC, Castle PE, et al. Five-year risk of cervical precancer following $\mathrm{p} 16 / \mathrm{Ki}-67$ dual-stain triage of HPV-positive women. JAMA Oncol 2019;5:181-6.

28 Baldur-Felskov B, Munk C, Nielsen TSS, et al. Trends in the incidence of cervical cancer and severe precancerous lesions in Denmark, 1997-2012. Cancer Causes Control 2015;26:1105-16.

29 Lei J, Ploner A, Lagheden C, et al. High-risk human papillomavirus status and prognosis in invasive cervical cancer: a nationwide cohort study. PLoS Med 2018;15:e1002666. 